\title{
MS-MLPA is a specific and sensitive technique for detecting all chromosome 11p15.5 imprinting defects of BWS and SRS in a single-tube experiment
}

\author{
Manuela Priolo ${ }^{* 1,4}$, Angela Sparago ${ }^{2,4}$, Corrado Mammi ${ }^{1}$, Flavia Cerrato ${ }^{2}$, Carmelo Laganà ${ }^{1}$ \\ and Andrea Riccio ${ }^{2,3}$
}

\author{
${ }^{1}$ Operative Unit of Medical Genetics, Hospital of Reggio Calabria Az. Ospedaliera Bianchi-Melacrino-Morelli, V Petrara \\ Reggio Calabria, Reggio Calabria, Italy; ${ }^{2}$ Dipartimento di Scienze Ambientali, Seconda Università di Napoli, \\ via Vivaldi 43, Caserta, Italy; ${ }^{3}$ Institute of Genetics and Byophisics A. Bozzati Traverso, Naples, Italy
}

Human chromosome 11p15.5 harbours a large cluster of imprinted genes. Different epigenetic defects at this locus have been associated with both Beckwith-Wiedemann syndrome (BWS) and Silver-Russell syndrome (SRS). Multiple techniques (Southern blotting, COBRA and microsatellite analysis) have been used so far to detect various DNA methylation abnormalities, uniparental disomies and copy number variations, which are characteristics of these two diseases. We have now evaluated a methylation-specific multiplex-ligation-dependent probe amplification assay (MS-MLPA) for the molecular diagnosis of BWS and SRS. Seventy-three samples derived from BWS- and SRS-affected individuals and 20 controls were analysed by conventional tests and MS-MLPA in blind. All cases that were found positive with conventional methods were also identified by MS-MLPA. These included cases with paternal UPD11, hyper- or hypomethylation at the Imprinting Centre 1 or Imprinting Centre 2 and rare 11 p15.5 duplications. In summary, this MS-MLPA assay can detect both copy number variations and methylation defects of the $11 \mathrm{p} 15.5$ critical region within one single experiment and represents an easy, low cost and reliable system for the molecular diagnostics of BWS and SRS.

European Journal of Human Genetics (2008) 16, 565-571; doi:10.1038/sj.ejhg.5202001; published online 23 January 2008

Keywords: MS-MLPA; Beckwith-Wiedemann syndrome; Silver-Russell syndrome; 11 p15.5

\section{Introduction}

Genomic imprinting is an epigenetic mechanism causing the expression of a minority of genes to be monoallelic and dependent on its gametic origin. ${ }^{1}$ Beckwith-Wiedemann syndrome (BWS) (MIM 130650) is a developmental disorder characterized by the occurrence of variably combined overgrowth, macroglossia, abdominal wall

*Correspondence: Dr M Priolo, Operative Unit of Medical Genetics, Hospital of Reggio Calabria Az. Ospedaliera Bianchi-Melacrino-Morelli, Via Malacrino, V Petrara Reggio, Reggio Calabria 89100, Italy.

Tel: + 39096539 7296; Fax: + 39096539 7350;

E-mail: prioloma@libero.it

${ }^{4}$ These authors have contributed equally to this work.

Received 5 September 2007; revised 30 October 2007; accepted 11 December 2007; published online 23 January 2008 defects and increased incidence of embryonal tumours caused by defective expression of imprinted genes located on chromosome $11 \mathrm{p} 15.5 .^{2}$ Silver-Russell syndrome (SRS; OMIM 180860) is a clinically and genetically heterogeneous disorder characterized by severe pre- and postnatal growth defect, variable facial dysmorphisms, limb or facial asymmetry and developmental delay. Although maternal uniparental disomy of chromosome 7 has been reported in $\sim 10 \%$ of SRS patients, molecular defects at $11 \mathrm{p} 15.5$ have been recently described as a major genetic disturbance in SRS, indicating that the dysregulation of 11p15.5 imprinted genes may cause both growthenhanced and growth-restricted phenotypes. ${ }^{3,4}$ Both BWS and SRS include mostly sporadic and a minority of familial cases. 
A $1 \mathrm{Mb}$ cluster of imprinted genes is present at chromosome 11p15.5. The cluster is functionally divided into two domains that are autonomously controlled by separate Imprinting Control Regions or Imprinting Centres (IC1 and IC2). These are CpG-rich regions that work under different mechanisms, but share as a common feature to be differentially methylated on the maternally and paternally derived chromosomes (differentially methylated regions (DMRs)). The distal IC (IC1 or H19 DMR) is a methylationsensitive chromatin insulator controlling the imprinting of two genes, Insulin-like growth factor 2 (IGF2) and H19. ${ }^{1,5}$ IGF2 is a paternally expressed fetal growth factor gene with an important role in cancer development, while H19 is a maternally expressed non-coding RNA with possible tumoursuppressor functions. By binding to the multi-zinc-finger protein CTCF, IC1 prevents the activation of IGF2 and allows that of $H 19$ by downstream enhancers on the maternal chromosome. Conversely, DNA methylation on the paternal chromosome prevents CTCF binding at IC1 and allows the enhancer-mediated activation of IGF2, being the $H 19$ promoter hypermethylated and silenced. The proximal IC (IC2 or KvDMR1) includes the promoter of the non-coding KCNQ1OT1 gene that is antisense transcribed with respect to the protein-coding gene KCNQ1. The IC2 sequence itself and/or the KCNQ1OT1 transcript mediate the silencing of several genes of the region including the growth inhibitor CDKN1C. ${ }^{1,6,7}$

A great number of BWS and SRS cases show molecular abnormalities in the 11p15.5 imprinted gene cluster. In the BWS, the most common defects are uniparental paternal disomy (UPD) at 11p15.5 loci, gain of methylation at IC1 and loss of methylation at IC2. ${ }^{2,8}$ All these anomalies result in excess of the genes expressed from the paternal chromosome and defect of the genes expressed from the maternal chromosome. Inherited deletions of the ICs have been reported in a small number of patients with BWS. ${ }^{9,10}$ Almost 35\% of SRS patients display IC1 hypomethylation or 11 p15.5 maternal duplications. These defects result in the downregulation of IGF2 and activation of H19. ${ }^{4,11,12}$ Conventional molecular testing for the IC1/IC2 methylation abnormalities, 11p15 UPD and copy number variations in BWS and SRS consisted of multiple and time-consuming Southern blotting and microsatellite assays. ${ }^{2,3,8}$ We evaluated MS-MLPA for the diagnosis of both BWS and SRS, and challenged it with previously routine tests.

\section{Materials and methods Patients}

We collected DNAs from perypheral blood leukocytes of 73 patients with clinical diagnosis of BWS or SRS. All the genetic analyses were performed after the informed consent had been obtained from the parents of the patients. The experimental plan was approved by the ethical committee of the Second University of Naples.

\section{Methylation analysis by conventional methods}

Analysis of IC1 and IC2 methylation by Southern blotting or COBRA and detection of $11 \mathrm{p} 15.5 \mathrm{UPD} /$ copy number variations by microsatellite analysis were performed as described previously. ${ }^{9,13}$ All the SRS patients have been previously excluded for maternal UPD7 by microsatellite analysis.

\section{MS-MLPA analysis}

The MS-MLPA probe mixture (ME030BWS/SRS) was purchased from the manufacturer (MRC-Holland, Amsterdam/ $\mathrm{NL}$ ). This mix contains 45 probes, 27 of which are specific for the BWS/SRS region in 11p15. 13 of the $11 \mathrm{p} 15$ probes are methylation-specific since they contain an HhaI recognition site. In particular, seven probes (five of which are methylation-specific) cover the $H 19$ gene and its differentially methylated $5^{\prime}$ region located inside IC1 (H19DMR). Four probes (three methylation-sensitive) cover the IGF2 gene and its DMR0 region. A further 14 probes (six methylation-sensitive) hybridize to the KCNQ1 gene, including the IC2 region (KvDMR). Finally, two probes (both methylation-insensitive) recognize CDKN1C (Figure 1a). We used a total of $200 \mathrm{ng}$ of genomic DNA extracted from peripheral blood by the salting-out procedure. $10 \mathrm{mmol} / \mathrm{l}$ Tris-EDTA (pH 8.0) was added to DNA if dilution was necessary. After $16 \mathrm{~h}$ of hybridization at $60^{\circ} \mathrm{C}$, samples were equally split into two aliquots. The first aliquot underwent ligation only, whereas the second one underwent ligation followed by enzymatic digestion with HhaI (a restriction enzyme recognizing only unmethylated DNA) and PCR amplification using universal primers. In the latter case, amplification products were obtained and detected by capillary electrophoresis only if the CpG included in the Hhal site was methylated. Ligation, enzymatic digestion, and PCR amplification were performed according to the manufacturer's instructions. All samples were run in duplicate and at least two normal samples were analysed in each MS-MLPA experiment, as controls. PCR products $(1 \mu \mathrm{l})$ were mixed with $0.4 \mu \mathrm{l}$ of internal size standard (500LIZ Genescan; Applied Biosystems) and $9 \mu$ l of deionized formamide, and injected into an ABI-3130 genetic analyzer (Applied Biosystems) equipped with a $36 \mathrm{~cm}$, four capillaries array using POP7 as polymer. The samples were injected for $20 \mathrm{~s}$ at $1.5 \mathrm{kV}$ and run for a total time of $1200 \mathrm{~s}$ at $15 \mathrm{kV}$.

\section{Data analysis}

The reliability and normal values for the probes of this MSMLPA kit were determined by testing 20 normal control DNA samples. A spreadsheet was developed in Microsoft ${ }^{\mathrm{TM}}$ Excel to process the sample data efficiently. The estimation of allelic copy number was performed by comparing the quantified PCR products of the probes in each undigested sample with those in other samples. Data were normalized by dividing the peak area of each probe by the average peak 


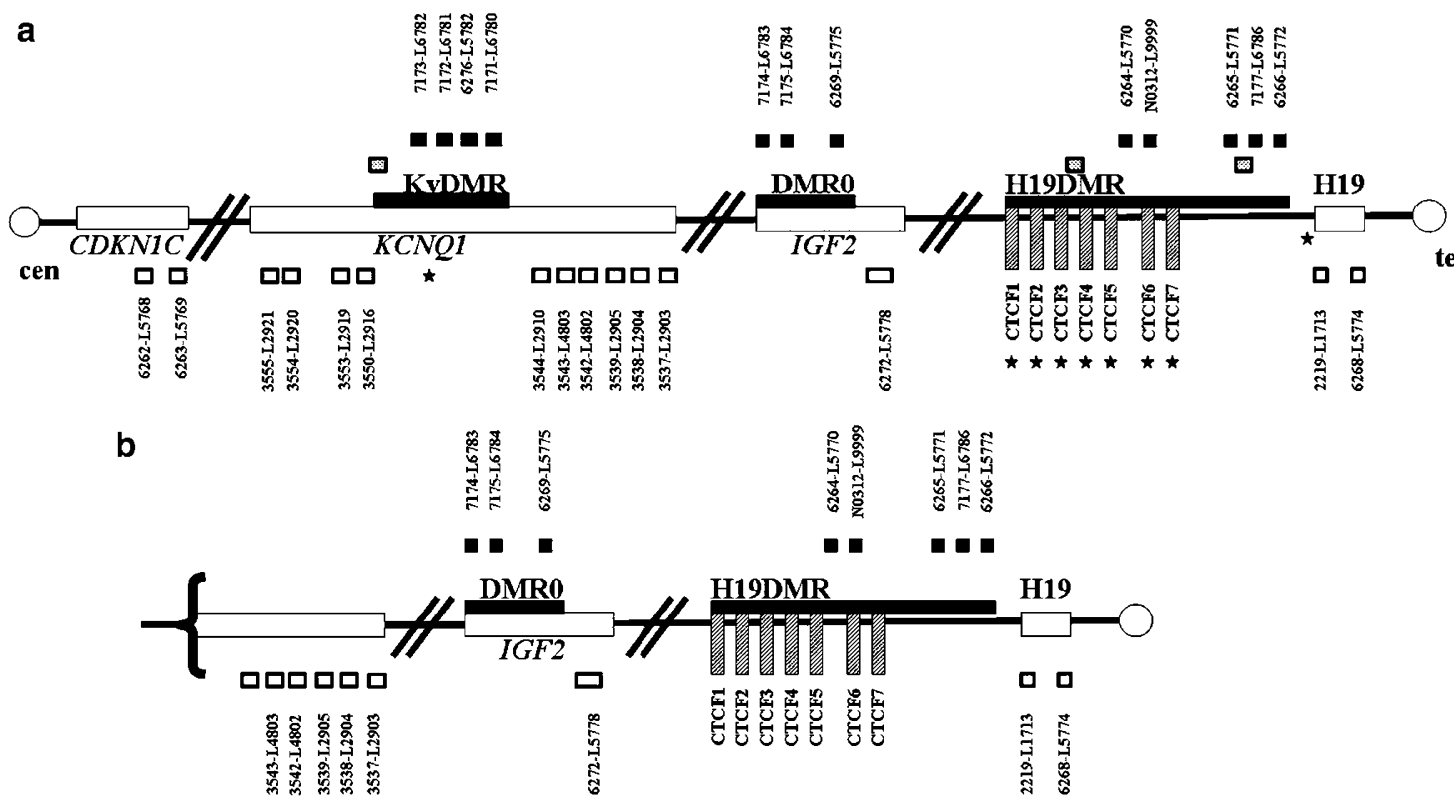

Figure 1 The $11 \mathrm{p} 15.5$ imprinted gene cluster and the MS-MLPA probes. (a) Schematic diagram of the genomic structure of the $11 \mathrm{p} 15.5$ region covered by the MS-MLPA probes used in this study (not in scale). Methylation-sensitive probes are presented in black boxes, other probes are presented in clear boxes. The position of the Southern blotting probes is indicated by hatched boxes. Asterisks show the restriction sites analysed by COBRA. Larger open rectangles indicate the position and extension of the imprinted genes, thick black lines indicate the extension of the DMRs and striped vertical rectangles the CTCF target sites. (b) Extension of a paternal duplication as determined by MS-MLPA in a BWS case. All probes corresponding to the duplicated sequences and their locations are shown.

Table 1 Molecular alterations of the BWS and SRS cases included in this study

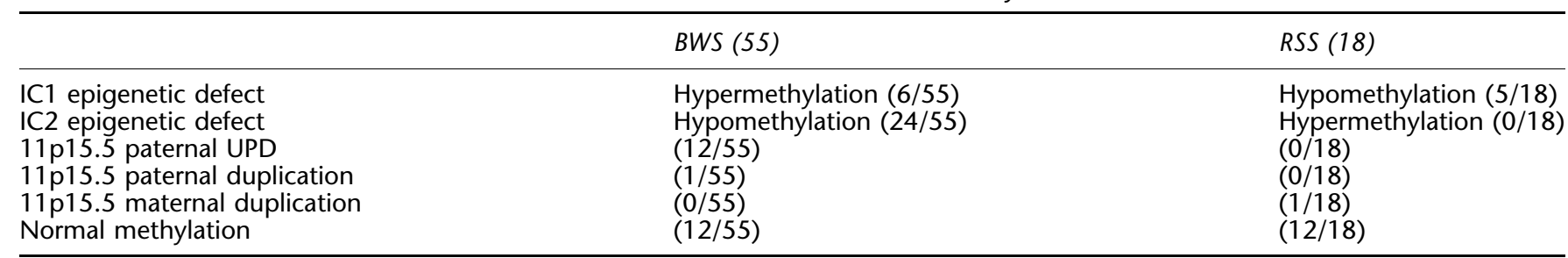

area of the same sample and then by the average peak pattern of all the samples run in the same experiment. ${ }^{14}$ Quantification of the methylation status of each analysed CpG was done by dividing the peak area of the relative methylation-sensitive probe by the combined peak areas of the nearest control probes lacking an HhaI site. The corrected peak height of each fragment in the digested sample was then divided by the corrected peak area of the corresponding fragment in the undigested sample. This number is referred as the 'methylation index'. To simplify the interpretation of data, we calculated the mean methylation index obtained with all the KvDMR probes (7173-L6782, 7172-L6781, 6276-L5782 and 7171-L6780) and that obtained with all the H19DMR probes (6264L577, 6265-L5771, 7177-L6786 and 6266-L5772) and indicated them as IC2 and IC1 methylation indices, respectively.

\section{Results}

A total of 55 DNA samples derived from individuals affected by BWS and 18 samples derived from individuals affected by SRS were analysed by Southern blotting or COBRA assays plus microsatellite analysis for the identification of $11 \mathrm{p} 15.5$ defects. The results are summarized in Table 1 . Out of 55 BWS patients, we found that 24 patients had IC2 hypomethylation (BWS-ICD2), 6 had IC1 hypermethylation (BWS-ICD1), 12 had paternal UPD at chromosome 11p15.5 (UPD11), 1 had paternal duplication of 11 p15.5 and 12 had none of the previous defects. Among the 18 SRS patients tested, 7 displayed IC1 hypomethylation (SRS-ICD1), 1 had maternal duplication of $11 \mathrm{p} 15.5$ and 10 none of the previous defects.

The same patients and 20 normal controls were blindtested by MS-MLPA. The analysis of the 20 control samples gave reproducible results with all the probes both for copy 
number and methylation, with the exception of the probe N0213-L9402, which (as also reported by the manufacturer) resulted to be non informative for the methylation analysis, because of a frequent polymorphism affecting the HhaI site (rs10732516). In particular, none of the 20 control samples had peak ratios $<0.75$ or $>1.35$ at two or more adjacent probes. The mean methylation indices for the normal samples were 0.52 (range $0.47-0.58$ ) for IC2 and 0.50 (range $0.46-0.55$ ) for IC1 (see Table 2). Standard deviation was 0.03 for both IC1 and IC2 methylation indices. IC2 methylation indices $<0.46$ were considered indicative of IC2 hypomethylation, IC1 methylation indices $>0.56$ indicative of IC1 hypermethylation and IC1 methylation indices $<0.44$ indicative of IC1 hypomethylation. The results obtained with the two methylation-sensitive probes specific for the IGF2 DMR0 region were inconsistent in some probes, indicating variable methylation of these CpGs in different individuals, and were therefore not taken into consideration for statistical analysis.

Table 2 summarizes the results obtained from the MSMLPA analysis of the BWS and SRS patients. All the molecular defects of the BWS cohort identified with the

Table 2 Summary of the MS-MLPA methylation indices in BWS and SRS

\begin{tabular}{|c|c|c|}
\hline & $\begin{array}{c}\text { IC2 } \\
\text { MI range }\end{array}$ & $\begin{array}{l}\text { IC1 } \\
\text { MI range }\end{array}$ \\
\hline Normal controls (20) & $0.47-0.58$ & $0.46-0.55$ \\
\hline Mean \pm SD & $0.52 \pm 0.03$ & $0.50 \pm 0.03$ \\
\hline BWS IC 2 hypomethylation $(24 / 55)$ & $0.00-0.40$ & $0.47-0.55$ \\
\hline BWS IC1 hypermethylation $(6 / 55)$ & $0.49-0.56$ & $0.63-0.97$ \\
\hline BWS paternal $11 \mathrm{p} 15.5$ UPD $(12 / 55)$ & $0.16-0.43$ & $0.62-0.79$ \\
\hline BWS Normal methylation $(10 / 55)$ & $0.48-0.58$ & $0.46-0.54$ \\
\hline SRS IC1 hypomethylation (7/18) & $0.47-0.56$ & $0.00-0.26$ \\
\hline SRS Normal methylation $(10 / 18)$ & $0.47-0.56$ & $0.48-0.55$ \\
\hline
\end{tabular}

${ }^{a}$ Anomalous methylation indices (MI) are outlined in bold. Standard deviations (SD) for both IC1 and IC2 methylation indices were calculated on 20 normal samples.

Figure 2 MS-MLPA peak patterns obtained with normal controls and BWS and SRS cases with different molecular defects. (a) normal sample, 'ligation only' reaction. (b) normal sample, 'digestion/ligation' reaction. (c) BWS sample with IC2 hypomethylation, digestion/ligation reaction. (d) BWS sample with IC1 hypermethylation, digestion/ ligation reaction. (e) BWS sample with paternal UPD11, digestion/ ligation reaction. (f) SRS sample with IC1 hypomethylation digestion/ ligation reaction. Grey arrows refer to the methylation-sensivite probes recognizing $I C 1$, black arrows refer to the methylation-sensitive probes recognizing IC2. Black stars indicate methylation control $(0 \%$ methylated) probes. Black arrowheads indicate the hypomethylated CpGs detected by IC2 probes in BWS-ICD2 and UPD samples. Grey arrowheads refer to the hypermethylated $\mathrm{CpGs}$ detected by IC1 probes in BWS-ICD1 and UPD11 samples. Grey arrowheads in panel $\mathrm{f}$ refer to hypomethylated $\mathrm{CpG}$ s detected by IC1 probes in SRS samples. Little black stars in panels $d$ and e on the first peak indicate the variable results obtained with the N0213-L9999 probe due to a sequence polymorphism. conventional methods were detected also with the MSMLPA assay. In particular, the 24 BWS-ICD2 samples showed IC2 methylation indices between 0.00 and 0.40 but IC1 methylation indices within normal range, the 6 BWS-ICD1 samples had IC1 methylation indices between 0.63 and 0.97 but IC2 methylation indices within the normal range and the 12 paternal UPD11 had IC2 methylation indices between 0.16 and 0.43 and IC1 methylation indices between 0.62 and 0.79 . Figure 2 shows
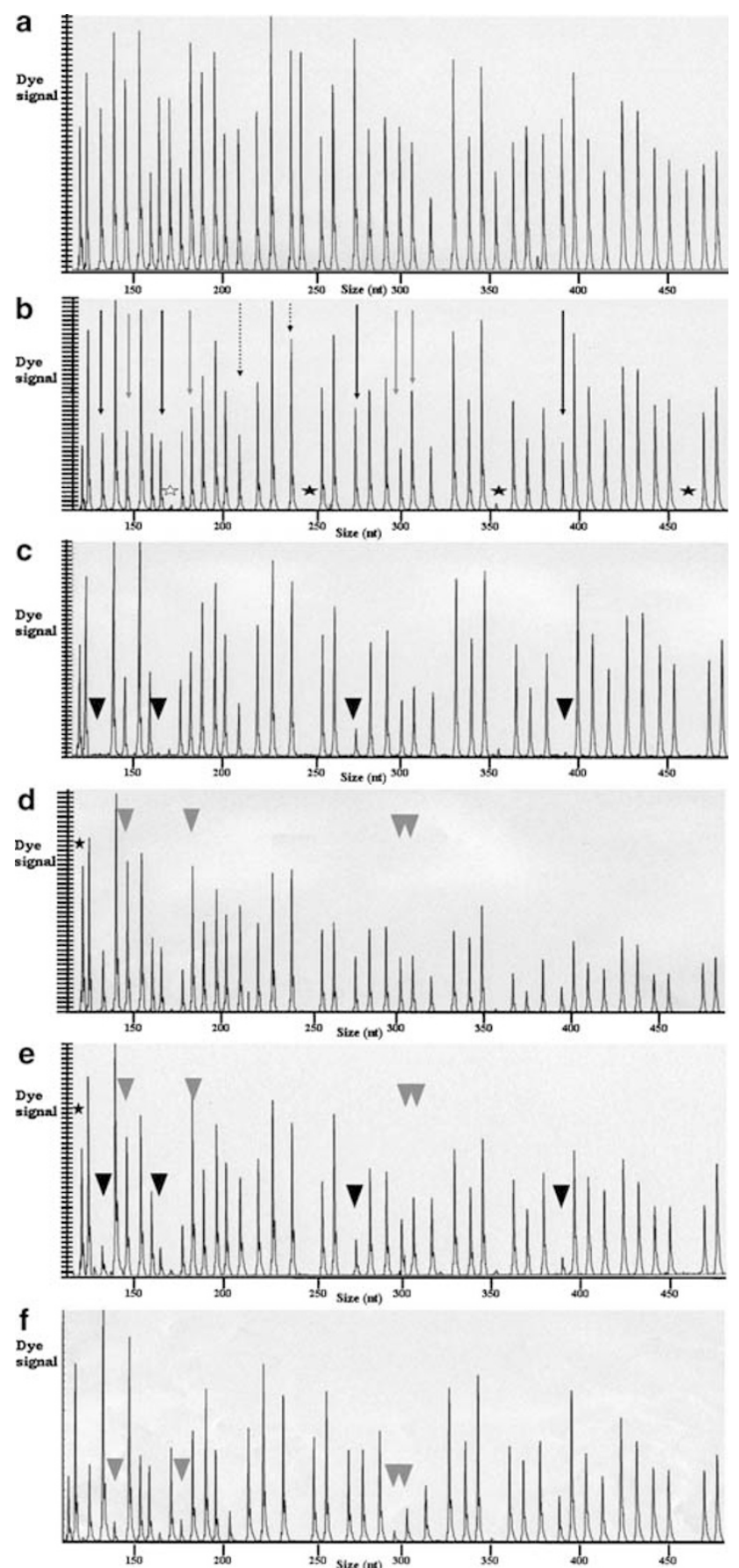
an example of the typical peak patterns obtained with normal controls (Figure 2a and b), BWS-ICD2 (Figure 2c), BWS-ICD1 (Figure 2d) and BWS-UPD11 (Figure 2e) samples. IC2 hypomethylation was indicated by abnormally low peaks corresponding to the four methylation-sensitive probes that were specific for KvDMR1 (7173-L6782, 7172L6781, 6276-L5782 and 7171-L6780), after HhaI digestion (see Figures 1a and 2c, black arrowheads). IC1 hypermethylation was indicated by abnormally high peaks corresponding to the four methylation-sensitive probes located inside the H19DMR (6264-L577, 6265-L5771, 7177-L6786 and 6266-L5772, see Figures 1a and 2d, grey arrowheads).

Interestingly, the MS-MLPA also detected those cases that had limited hyper- or hypomethylation. For instance, a sample with $60-70 \%$ methylation at IC1, as measured by COBRA, showed an IC1 methylation index of 0.68 by MSMLPA, while a sample with $30-40 \%$ methylation at IC2, as measured by COBRA, had an IC2 methylation index of 0.38 (Figure 3). In contrast, the 12 BWS samples in which no methylation defect had been identified with the conventional methods showed methylation indices within normal range with the MS-MLPA assay (Table 2).

Hypomethylation at IC1 was demonstrated by Southern blotting or COBRA and clearly confirmed by MS-MLPA in seven cases of our SRS cohort $(N=18)$. IC1 methylation indices were in the range of $0-0.26$, while IC2 methylation indices were within normal range in these cases (Table 2). In contrast, the 10 SRS samples in which no methylation defect had been identified with the conventional methods showed both IC1 and IC2 methylation indices within the normal range with the MS-MLPA assay (Table 2). Figure $2 \mathrm{f}$ shows the typical peak pattern obtained with the SRS cases with IC1 hypomethylation. The methylation defect was indicated by abnormally low IC1-specific peaks (probes 6264-L577, 6265-L5771, 7177-L6786 and 6266-L5772, see Figures $1 \mathrm{a}$ and $2 \mathrm{f}$, grey arrowheads).

The chromosome 11p15.5 duplications identified by Southern blotting and microsatellite analyses in a BWS patient and an SRS patient were also detected by the MSMLPA assay. In both cases, the peak area ratios for the 11 15.5 probes were $>1.35$ in the 'ligation only' reactions. In particular, the MLPA demonstrated that the BWS sample had only a partial duplication of the $11 \mathrm{p} 15.5$ region, as the probes centromeric to 3544-L2910 produced peak area ratios within the normal range (Figure $1 b$ ). Therefore, only the IC1 domain and the part of the IC2 domain telomeric to KvDMR1 appeared to be duplicated in this sample. For this BWS case, the 'ligation-digestion' reaction demonstrated an IC1 methylation index of 0.70 and an IC2 methylation index of 0.48 , indicating IC1 hypermethylation and normal IC2 methylation, as expected from a paternally inherited duplication of the IC1 domain. In the SRS sample, the ligation-only reaction showed a duplication covering all the analysed $11 \mathrm{p} 15.5$ region and the ligation-digestion reaction demonstrated an IC1 methyla-

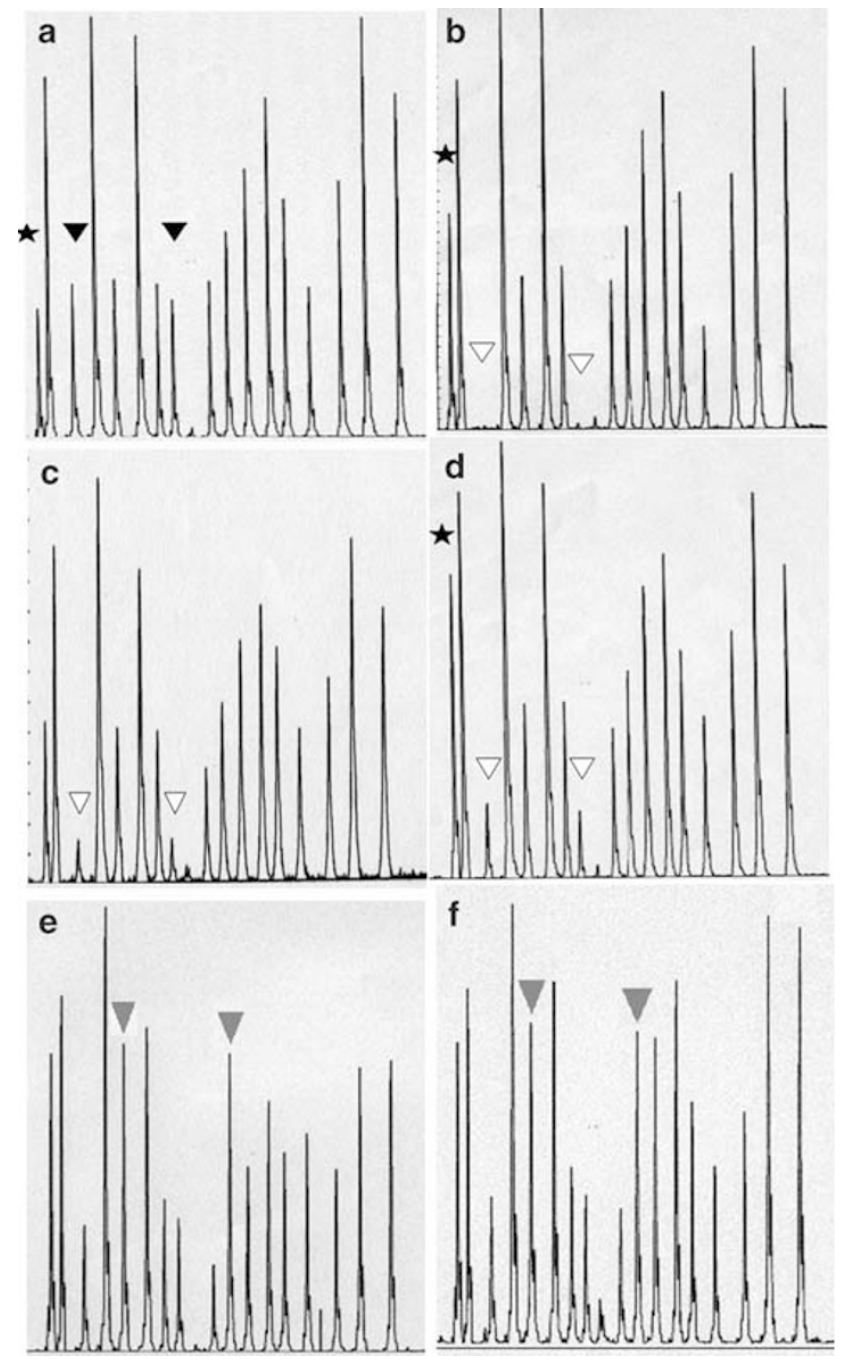

Figure 3 MS-MLPA peak patterns (partial view) obtained with normal controls or BWS patients with different degree of IC2 hypomethylation and IC1 hypermethylation. (a) normal sample, 'digestion/ligation' reaction (b) BWS sample with $2 \%$ IC2 methylation, digestion/ligation reaction (IC2 methylation index $=0$ ) (c) BWS sample with $28 \%$ IC2 methylation, digestion/ligation reaction. (IC2 methylation index $=0.16$ ) (d) BWS sample with $44 \%$ IC2 methylation, digestion/ligation reaction (IC2 methylation index $=0.38)(\mathrm{e})$ BWS sample with $98 \%$ IC1 methylation, digestion/ligation reaction (IC1 methylation index $=0.97$ ). (f) BWS sample with $75 \%$ IC1 methylation, digestion/ligation reaction (IC1 methylation index $=0.70$ ). DNA methylation levels were measured by Phosphorlmager (Molecular Dynamics) quantification of COBRA bands, while methylation indices were calculated from the peak areas of the MS-MLPA as described in the Materials and methods section. Black arrowheads indicate the peaks corresponding to two IC2 CpGs (probes 7173-L6782 and 6276L5782) with normal $50 \%$ methylation. White arrowheads indicate the peaks corresponding to the same CpGs in the BWS-ICD2 samples. Grey arrowheads refer to two IC1 CpGs (probes 6264-L577 and 6265L5771); Black stars indicate the variable results obtained with the N0213-L9999 probe.

tion index of 0.26 and an IC2 methylation index of 0.71 , indicating IC2 hypermethylation/IC1 hypomethylation, as expected from a maternally transmitted duplication. 
In conclusion, the results obtained with this MS-MLPA assay demonstrate that this method is a specific and very sensitive method, as efficient as combined Southern blotting, COBRA and microsatellite analyses, for identifying methylation defects, UPD and copy number variations at chromosome 11p15.5.

\section{Discussion}

The molecular defects of BWS and SRS are very heterogenous. Many of these abnormalities consist of epigenetic or copy-number alterations inside the $11 \mathrm{p} 15.5$ imprinted gene cluster. The identification of these defects usually requires the use of multiple assays. We evaluated the MSMLPA for the molecular diagnosis of BWS and SRS and challenged it with previous routinely used tests. The results obtained demonstrated that MS-MLPA is a sensitive and reliable tool for the identification of most of the molecular defects of BWS and SRS.

Conventional techniques, such as the Southern blotting assay, require a large amount of genomic DNA and multiple hybridization experiments for the analysis of DNA methylation at multiple loci. Alternative methods, such as COBRA or methylation-specific PCR, are quite laborious to standardize and are not suitable for routine molecular diagnostics. In addition, these assays do not provide accurate information on copy number variations, which are better identified by microsatellite or FISH analyses. MS-MLPA, needs very little amount of DNA $(20-200 \mathrm{ng})$, is very rapid and provides information on copy number and methylation status of multiple loci in a single-tube experiment. ${ }^{15}$

Most of the epigenetic defects of BWS and SRS occur sporadically and are present in the mosaic form in the affected individuals. $^{2,3}$ It is therefore important to use a sensitive diagnostic method to identify all the mosaic cases. We proved that the MS-MLPA assay described is not only very accurate but also extremely sensitive, concerning the detection of $11 \mathrm{p} 15.5$ defects. In fact, this method correctly identified also BWS cases with 60-70\% IC1 methylation (methylation index 0.68) and BWS cases with $30-40 \%$ IC2 methylation (methylation index 0.38).

A great advantage of MS-MLPA is that this method concomitantly determines both copy number and methylation level of the investigated loci. This is clearly demonstrated by the results we obtained on two cases with $11 \mathrm{p} 15.5$ duplication. While the ligation-only reaction demonstrated the copy number increase, the ligationdigestion reaction provided information on the parental origin of the duplicated region. The methylation indices of the DMRs indicated that the former case was a BWS sample with a paternally inherited 11p15.5 duplication and the latter was an SRS sample with maternal duplication of this chromosome region. These results were confirmed by the microsatellite analysis. Interestingly, the MS-MLPA results demonstrated that, in the BWS case, the duplication did not include KvDMR and the centromeric part of domain 2. As expected, the sample showed isolated IC1 hypermethylation.

While revising this article, an independent group $^{16}$ reported on the use of MS-MLPA for the analysis of chromosome $11 \mathrm{p} 5.5$ defects obtaining similar results as ours and further confirming that MS-MLPA can be considered as a robust assay for the analysis of this region.

Although we proved that MS-MLPA assay is very useful for the analysis of BWS and SRS, we believe that the method can be further improved in the future by modifying some of the probes. For instance, methylation-sensitive probes located in the more centromeric part of the H19DMR could be useful to detect the IC1 microdeletions present in a subset of the BWS patients. ${ }^{9,13}$ On the other hand, the H19 DMR-specific probe (N0213-L9999) corresponding to a common SNP could be omitted. In conclusion, our experience with this large cohort of patients indicate that the MS-MLPA represents an easy, low cost and reliable system for the molecular diagnostics of BWS/SRS cases with either imprinting defects and duplications at chromosome 11p15. Paternal UPD11 can also be identified, although microsatellite testing should be offered to confirm these cases.

\section{Acknowledgements}

We thank all the patients and their families for their participation to the study. This work was supported by grants from MIUR PRIN 2005, Associazione Italiana Ricerca sul Cancro, Istituto Superiore di Sanità and Telethon, Italia Grant no. GGP04072 (to A.R.). FC was recipient of a fellowship from Società Italiana di Cancerologia and Fondazione Pezcoller.

\section{References}

1 Edwards CA, Ferguson-Smith AC: Mechanisms regulating imprinted genes in clusters. Curr Opin Cell Biol 2007; 19: 281-289.

2 Weksberg R, Shuman C, Smith AC: Beckwith-wiedemann syndrome. Am J Med Genet C Semin Med Genet 2005; 137: 12-23.

3 Smith AC, Choufani S, Ferreira JC, Weksberg A: Growth regulation, imprinted genes, and chromosome 11p15.5. Ped Research 2007; 61: 43R-47R.

4 Gicquel C, Rossignol S, Cabrol S et al: Epimutation of the telomeric imprinting center region on chromosome $11 \mathrm{p} 15$ in Silver-Russell syndrome. Nat Genet 2005; 37: 1003-1007.

5 Hark AT, Schoenherr CJ, Katz DJ et al: CTCF mediates methylation-sensitive enhancer-blocking activity at the H19/Igf2 locus. Nature 2000; 405: 486-489.

6 Smilinich NJ, Day CD, Fitzpatrick GV et al: A maternally methylated CpG island in KvLQT1 is associated with an antisense paternal transcript and loss of imprinting in Beckwith-Wiedemann syndrome. Proc Natl Acad Sci USA 1999; 96: 8064-8069.

7 Mancini-Dinardo D, Steele SJ, Levorse JM, Ingram RS, Tilghman SM: Elongation of the Kcnq1ot1 transcript is required for genomic imprinting of neighboring genes. Genes Dev 2006; 20: $1268-1282$. 
8 Cooper WN, Luharia A, Evans GA et al: Molecular subtypes and phenotypic expression of Beckwith-Wiedemann syndrome. Eur J Hum Genet 2005; 13: 1025-1032.

9 Sparago A, Cerrato F, Vernucci $M$ et al: Microdeletions in the human H19 DMR result in loss of IGF2 imprinting and Beckwith-Wiedemann. Nat Genet 2004; 36: 958-960.

10 Niemitz EL, DeBaun MR, Fallon J et al: Microdeletion of LIT1 in familial Beckwith-Wiedemann syndrome. Am J Hum Genet 2004; 75: 844-849.

11 Bliek J, Terhal P, van den Bogaard M-J et al: Hypomethylation of the H19 gene causes not only Silver-Russell syndrome (SRS) but also isolated asymmetry or an SRS-like phenotype. Am J Hum Genet 2006; 78: 604-614.

12 Eggermann $\mathrm{T}$, Meyer E, Schönherr $\mathrm{N}$ et al: Epigenetic mutations in $11 \mathrm{p} 15$ in Silve-Russell syndrome are restricted to the telomeric imprinting domain. J Med Genet 2006; 46: 615-616. 4.
13 Sparago A, Russo S, Cerrato F et al: Mechanisms causing imprinting defects in familial Beckwith-Wiedemann syndrome with Wilms' tumour. 2007; 16: 254-264.

14 Koolen DA, Nillesen WM, Versteeg MHA et al: Screening for subtelomeric rearrangements in 210 patients with unexplained mental retardation using multiplex ligation dependent probe amplification (MLPA). J Med Genet 2004; 41: 892-899.

15 Nygren AO, Ameziane N, Duarte HM et al: Methylation-specific MLPA (MS-MLPA): simultaneous detection of CpG methylation and copy number changes of up to 40 sequences. Nucleic Acis Res 2005; 16: e128.

16 Scott RH, Douglas J, Baskcomb L et al: Methylation-specific MLPA (MS-MLPA) robustly detects and distinguishes $11 \mathrm{p} 15$ abnormalities associated with overgrowth and growth retardation. $J$ Med Genet 2007: e-pub ahead of print 15 October 2007. 